\title{
Luminescence from acoustic-driven laser-induced cavitation bubbles
}

\author{
Claus-Dieter Ohl* \\ Drittes Physikalisches Institut, Universität Göttingen, Bürgerstraße 42-44, D-37073 Göttingen, Germany
}

(Received 20 July 1999)

\begin{abstract}
The influence of a continuous sound field on the first oscillation cycle and on the cavitation luminescence of a transient laser-induced bubble is investigated experimentally. The variation of the collapse phase is predicted with a simple numerical model and compared with experiment. Bubble dynamics is mainly influenced by three parameters: the phase of bubble generation, the size of the bubble, and the amplitude of the sound field. The experimentally found enhancement and reduction of the luminescence is discussed and several suggestions are made for further boosting of the collapse strength.
\end{abstract}

PACS number(s): 78.60.Mq, 47.55.Bx, 47.55.Dz

A vast concentration of energy occurs during the collapse of a cavitation bubble. The mostly spherical liquid flow is responsible for the compression and rapid heating of the bubble interior, giving rise to shock wave emission into the liquid phase and radiation of visible light at the time of maximum compression. This simple but effective mechanical system thus attracts research with the aim of enhancing the bubble collapse to achieve physical conditions inside the bubble that might allow nuclear fusion to take place. Nigmatulin et al. [1] and Moss et al. [2] have proposed different techniques for enhancement and two patents describe the construction of nuclear fusion cells [3]. Holzfuss et al. [4] succeeded in increasing the luminescence from a single stable oscillating bubble [5] (commonly abbreviated SBSL) by using second harmonic driving. However, the need to levitate a bubble by the same sound field may limit the amount of enhancement possible by this approach.

An approach by which the collapse of a large transient cavitation bubble can be enhanced is presented. Generally, a transient cavitation bubble grows explosively with rapid cooling of its interior, expanding to a maximum bubble size, largely due to the liquid inertia, and collapses violently with the emission of shock waves and light. Transient cavitation bubbles can be produced in a variety of ways [6], such as by explosives, electrical discharges, neutrons, and the focusing of intense light. These bubbles oscillate only a few times before (surface) instabilities break them up. In this article we investigate the effects of an imposed sound field on transient cavitation bubbles. With a simple numerical model, we study the alteration of their dynamics and present an experimental setup for laser-induced cavitation bubbles. In particular, we present experimental results for the duration of the first oscillation cycle and the energy of the light emitted upon collapse.

The nucleation of a transient cavitation bubbles is a very complex phenomenon, which involves energy exchange during the early expansion stage followed by a rapid nonequilibrium cooling of the bubble interior [7]. These complications are handled, starting with a bubble that already has

\footnotetext{
*Present address: Department of Fluid Dynamics and Heat Transfer, University of Twente, P.O. Box 217, 7500 AE Enschede, The Netherlands.
}

some energy. The model starts at time $t=t_{0}$ with a bubble of radius $R\left(t=t_{0}\right)$ and a bubble wall velocity of $\dot{R}\left(t=t_{0}\right)$. The bubble interior is in thermal equilibrium with the liquid. These are the initial conditions for an ordinary differential equation describing the spherical bubble dynamics. We use the Gilmore model [8], taking into account compressibility effects of the liquid:

$$
\begin{gathered}
\left(1-\frac{\dot{R}}{C}\right) R \ddot{R}+\frac{3}{2}\left(1-\frac{\dot{R}}{3 C}\right) \dot{R}^{2} \\
=\left(1+\frac{\dot{R}}{C}\right) H+\left(1-\frac{\dot{R}}{C}\right) \frac{R}{C} \frac{d H}{d t}, \\
H=\int_{P_{\infty}}^{p(R)} \frac{d p}{\rho}, \frac{p(R, \dot{R})+B}{P_{0}+B}=\left(\frac{\rho}{\rho_{0}}\right)^{n}, \\
C=\left.c\right|_{r=R}=\left.\sqrt{\frac{d p}{d \rho}}\right|_{r=R}=\sqrt{c_{0}^{2}+(n-1) H}, \\
p(R, \dot{R})=\left(P_{0}+\frac{2 \sigma}{R_{0}}\right)\left(\frac{R_{0}^{3}-a^{3}}{R^{3}-a^{3}}\right)-\frac{2 \sigma}{R}-\frac{4 \mu}{R} \dot{R},
\end{gathered}
$$

where $R$ is the bubble radius, $R_{0}$ represents the equilibrium bubbles and is taken to be constant for $R_{\max }^{n s} / R_{0}=9.1$ [9], and $c, \rho, p$ are the speed of sound in the liquid, its density, and the pressure in the liquid at the bubble wall, respectively. The pressure at infinity is $P_{\infty}=P_{0}+P_{a}(t)$, where $P_{a}(t)$ is the imposed acoustic driving term. We assume a sinusoidal driving $P_{a}=p_{A} \sin (2 \pi \nu t+\phi)$, where $p_{A}$ is the amplitude and $\phi$ the phase. $H$ is the enthalpy difference between the bubble wall and infinity. Numerical constants appearing in Eq. (1) are $c_{0}=1480 \mathrm{~m} / \mathrm{s}, \rho_{0}=998 \mathrm{~kg} / \mathrm{m}^{2}, \quad P_{0}=1.013$ bar, $\sigma$ $=0.07275 \mathrm{~N} / \mathrm{m}, \mu=0.001 \mathrm{~N} \mathrm{~s} / \mathrm{m}^{2}, \quad n=7, B=3122$ bar. The relation $a=R_{0} / 9.174$ refers to a Van der Waals hardcore [10] for water vapor. The mass flux by rectified diffusion has been shown to be small and is neglected [11].

In the absence of a sound field the bubble grows to a maximum radius $R_{\max }^{n s}$. The velocity necessary to reach this radius, neglecting all dissipation mechanisms, can be calculated from [12] 

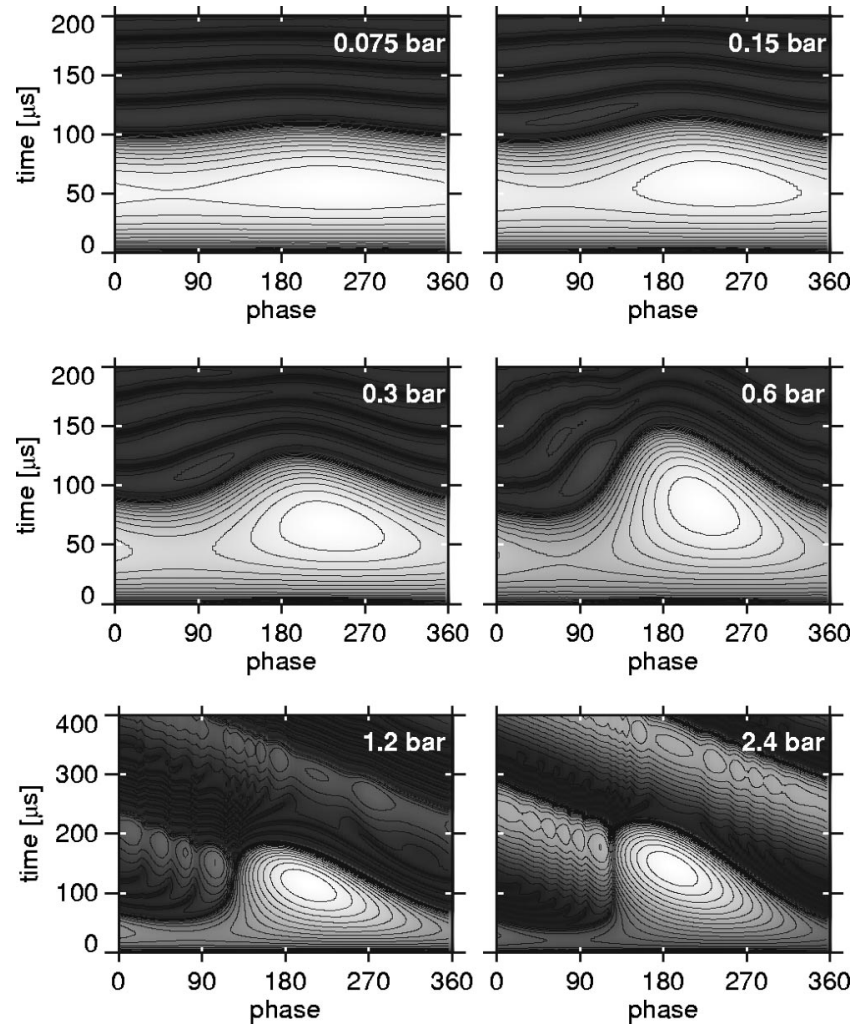

FIG. 1. The temporal response ( $y$ axes) of a bubble of radius $R(t)$ for different pressure amplitudes $p_{A}$, printed in each frame, is plotted with respect to its dependence on the initial phase lags. In each frame the radius of the bubble is depicted linearly with gray shades, where dark shades are small bubble radii and white shades the maximum bubble size. For easier reading, contour lines are overlayed connecting bubble radii of the same size. The driving frequency is $4.5 \mathrm{kHz}$, the initial radius $R\left(t_{0}\right)=64 \mu \mathrm{m}$, and initial bubble wall velocity $U\left(t_{0}\right)=255 \mathrm{~m} / \mathrm{s}$ leading to a maximum bubble radius of $R_{\max }^{n s}=0.58 \mathrm{~mm}$.

$$
\dot{R}\left(t=t_{0}\right)=\sqrt{\frac{2 P_{0}}{3 \rho}\left[\left(\frac{R_{\max }^{n s}}{R\left(t=t_{0}\right)}\right)^{3}-1\right]},
$$

which is in good agreement with the full numerical solution of Eq. (1) for cases investigated here. The first expansioncollapse cycle lasts about $2 T_{C}^{n s}$ where the Rayleigh's collapse time [12], $T_{C}^{n s}$, is the time that it takes for an empty cavity to shrink from an initial radius $R_{\max }^{n s}$ to zero size:

$$
T_{C}^{n s} \approx 0.91 R_{\max }^{n s} \sqrt{\frac{\rho}{P_{0}}} .
$$

In the presence of a sound field the bubble dynamics is different. The range of frequencies, $\nu$, in which the collapse is enhanced can be deduced approximately from the collapse time $T_{C}^{n s}$. The bubble expands during the low pressure and collapses at the high pressure phase of the sound field; therefore,

$$
\left(4 T_{C}^{n s}\right)^{-1} \leqslant \nu \leqslant\left(2 T_{C}^{n s}\right)^{-1}
$$

However, the sound field prolongs the expansion stage and shortens the collapse phase in an uneven way [13]. Hence, Eq. (4) is only valid for small driving pressures. For



FIG. 2. Experimental arrangement for the acoustic driving of a laser induced cavitation bubble. (a) Overview. (b) Side view of the cuvette, perpendicular to the direction of the laser beam, depicting the position of the cavitation bubble.

example, the estimated driving frequency for enhancement is in between 4.5 and $9.2 \mathrm{kHz}$ for a typical laser induced cavitation bubble of $R_{\max }^{n s}=0.6 \mathrm{~mm}$.

Each frame of Fig. 1 shows the calculated growth and collapse with a varying phase lag $\phi$ according to Eq. (1). The amplitude $p_{A}$ of the sound field doubles in the frames from left to right and from top to bottom. Up to a moderate acoustic pressure $(0.6$ bar) the expansion stage exhibits only a small dependence on the phase lag, such that the contour lines during this time are almost horizontal. Later in time the liquid slows down and the sound field influences the bubble dynamics. Hence, the bubble radius $R(t)$ depends on the phase. This is the reason for the curvature of the contour lines. The first cycle shortens or lengthens according to the action of the sound field. For small acoustic amplitudes the phase lag between minimum and maximum bubble expansion is approximately $180^{\circ}$, decreasing to $90^{\circ}$ at higher forcing pressures. The maximum bubble radius and the duration of the second cycle may be taken as indicators of a strong collapse. A high bubble wall velocity during the collapse leads to a strong damping by acoustic radiation, thus decreasing the duration of the second oscillation cycle. For small and medium pressures, enhanced light emission is to be expected at a phase lag of approximately $\phi=200^{\circ}$.

Figure 2(a) shows a sketch of the experimental arrangement. The transient cavitation bubble is produced by focusing a single light pulse from an infrared $Q$-switched $\mathrm{Nd}$ :YAG laser with a pulse width (FWHM) of $8 \mathrm{~ns}$ and a pulse energy, after suitable filtering of the light with neutral glass filters, between 1.8 and $3 \mathrm{~mJ}$ into bidistilled water. Two aberration-corrected lenses [15] (L1 with focal distance $f$ $=82 \mathrm{~mm}$ and L2, $f=16 \mathrm{~mm}$ ) allow for a nearly spherical focal area where the intensity is high enough to produce a plasma which, after recombination, becomes a rapidly expanding vapor bubble. The laser energy is monitored and recorded for further analysis on a sampling oscilloscope 


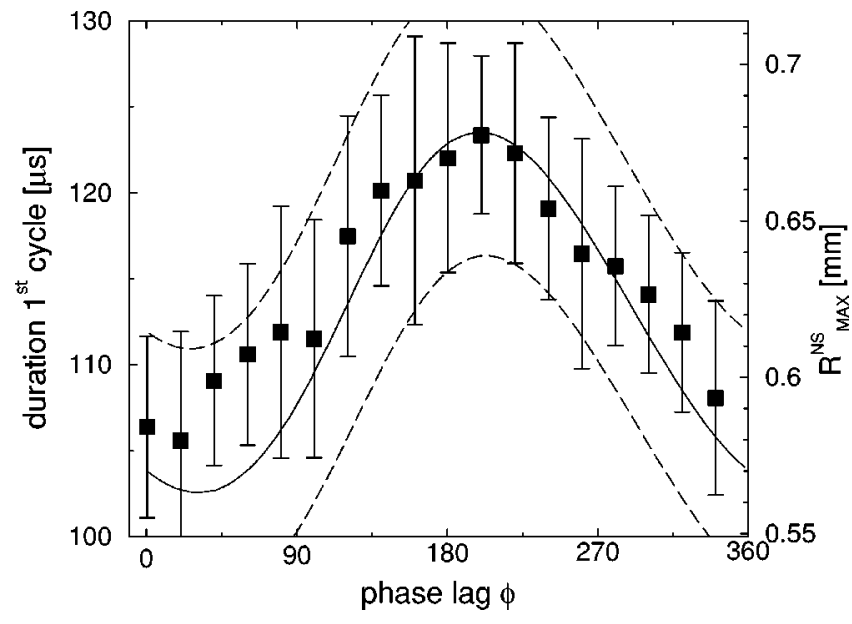

FIG. 3. Variation of the bubble life time $\left(2 T_{X}\right)$ by the sound field at a frequency $\nu$ of $4.5 \mathrm{kHz}$ and an amplitude $p_{A}$ of 0.15 bar in dependence on the phase lag $\phi$. The experimental data points ( $\boldsymbol{\square})$ depict the average over 150 experimental runs and the error bars indicate the standard deviation. The dashed and solid curves are from the model (see text). The corresponding maximum bubble radius from Eq. (3) is given on the right axis.

(DSO 1). For data analysis, bubbles are considered only when the laser energy deviates by less than $\pm 3 \%$ of the mean laser energy.

A principal element of this experimental setup is a piezo ceramic (PZT) transducer, a $5 \mathrm{~mm}$ thick hollow cylinder with an outer diameter of $65 \mathrm{~mm}$ and a length of $70 \mathrm{~mm}$. Two holes $10 \mathrm{~mm}$ in diameter have been drilled on opposing sides of the cylinder. One hole is used to inject the laser pulse into the cylinder, and the second hole is used to insert a hydrophone for measuring the amplitude and phase of the sound pressure at the focal region. To prevent corrosion and for electrical isolation purposes the cylinder is coated with PVC paint. The cylinder is submerged in a cuvette. A tube amplifier (Rhode and Schwarz) takes a sinusoidal signal from a function generator and drives the transducer at frequencies from 4 to $7 \mathrm{KHz}$. The distance between the focal region of the laser and the inner wall of the transducer, being the closest boundary to the bubble [16], is $20 \mathrm{~mm}$. The phase and the acoustic pressures are measured with a calibrated hydrophone (8103, Brüel and Kjaer).

The maximum repetition rate of the experiment is approximately $1 \mathrm{~Hz}$. After $1 \mathrm{~s}$ bubbles are not visible near the the focal area and therefore are not likely to influence the dynamics of a newly produced bubble. To maintain a precise phase lag between the function generator and the laser at this low repetition rate, the sync-out signal of the function generator is delayed and divided in frequency with a programmable delay generator (DG 535, Stanford Research). Upon receiving a trigger, this delay generator fires the laser after an adjustable delay and remains in a busy mode for $1 \mathrm{~s}$, not responding to further trigger events from the function generator. The focal area is imaged with the lens L3 ( $f$ $=50 \mathrm{~mm}$ ) onto the entrance window of a photomultiplier (R5600-3, Hamamatsu). A shortpass filter (F1) is placed in front of the photomultiplier (PMT) to protect it from scattered laser light.

Two light emission events are recorded. The first one is due to the laser induced dielectric breakdown causing a con-

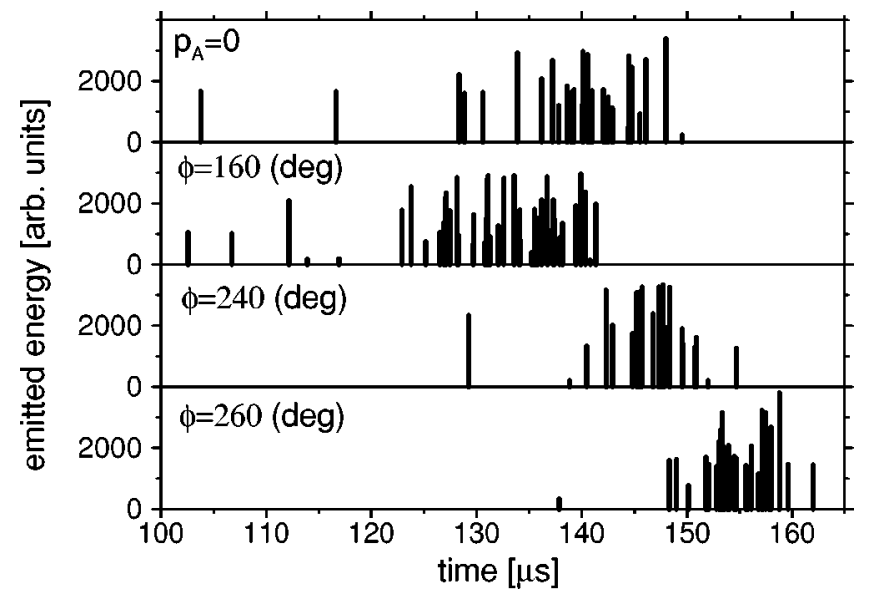

FIG. 4. Time of the luminescence and the emitted energy for four cases: top, in the absence of a sound field; below, for three different phase lags, $\phi$ with an acoustic pressure $p_{A}=0.45$ bar and frequency $\nu=6.3 \mathrm{kHz}$.

tinuum emission. The second light emission, the single cavitation bubble luminescence (SCBL), occurs at the time of maximum compression of the bubble interior [17]. The duration of the first expansion and collapse cycle is the time span between these two emission events. The signal of the PMT is converted and amplified into a voltage signal and fed into the second sampling oscilloscope DSO2 (TDS 784A, Tektronix). The DSO2 has enough memory to record, in real time, both emission events at a sampling rate of $1 \mathrm{GHz}$. The emitted energy at collapse, assuming isotropic radiation and a constant spectral distribution, is proportional to the integral over the SCBL signal. For high pressure, low frequency driving, a long data acquisition time and the large amount of samples are necessary to run the experiment in a stand-alone fashion by means of a computer.

Figure 3 displays the measured duration of the first oscillation cycle for 18 different phase lags at a driving pressure of $p_{A}=0.15$ bar. In the absence of the sound field, the bubble collapses at $114.6 \mu \mathrm{s}$. Due to the statistical nature of the bubble generation [14] process, there is a large jitter in the duration of the first cycle. Figure 3 shows the averaged values with their standard deviations from 150 experimental runs.

We find an increase of $17 \%$ in the duration of the first cycle from $\phi=20^{\circ}$ to $\phi=200^{\circ}$. The solid line is the calculated duration (see Fig. 1, $p_{A}=0.15$ bar) and shows good agreement with the observed duration. The dashed lines in Fig. 3 indicate the influence of the jitter on bubble sizes: the upper curve for an increased and the lower curve for a decreased $R_{\max }^{n s}$ using the measured jitter of $\pm 7.9 \mu \mathrm{s}$ (in absence of a sound field). All experimental data points lie well within these upper and lower bounds. This experiment clearly shows the increase and decrease of the duration of the first oscillation cycle as a result of a relatively small acoustic forcing pressure, compared to the ambient pressure.

We investigate the influence of the sound field on the emitted energy for a higher driving pressure $p_{A}=0.45$ bar at a frequency of $\nu=6.3 \mathrm{kHz}$. In Fig. 4 different temporal distributions of the emitted energy of the SCBL are plotted; in the top row no sound field is present, and below three distributions at different phase lags $\phi$ are given. The distribution 




FIG. 5. Comparison of the averaged duration of the first cycle (a) and the averaged emitted energy (b) with $p_{A}=0.45$ bar and $\nu$ $=6.3 \mathrm{kHz}$. The horizontal lines depict the averaged value in the absence of a sound field.

in the absence of sound, top row of Fig. 4, narrows and its center of mass shifts when the phase of the sound field is varied. The averaged duration of the first cycle for 18 phase lags $\phi$ and the averaged SCBL energies are shown in Figs. 5(a) and 5(b), respectively.

The duration of the first cycle is varied by $19 \%$ from $\phi$ $=160^{\circ}$ to $\phi=260^{\circ}$, and a comparison with the model shows agreement with the maximum response. However, the acoustic driving signal in the experimental run deviates from a pure sine. Therefore, the model (not shown) predicts a slightly stronger decrease of the first cycle duration for the phases ranging from $60^{\circ}$ to $160^{\circ}$. The averaged emitted en- ergy, Fig. 5(b), increases overall with increased duration of the first cycle $240^{\circ} \leqslant \phi \leqslant 360^{\circ}$. The emitted energy varies from the weakest emission $\phi=120^{\circ}$ to the strongest $\phi$ $=240^{\circ}$ by $90 \%$. As the averaged light emission possesses a maximum at $240^{\circ}$, the duration of the first cycle reaches its maximum at a later phase lag $\left(\phi=260^{\circ}\right)$. One reason for this behavior might be the increased influence of the rigid boundary (the transducer wall) as the bubble gets bigger inducing a slightly more asymmetric collapse which leads to a less effective energy concentration upon collapse [16].

We conclude that the dynamics and the emitted light energy from large transient cavitation bubbles can be altered by an additional sound field. We find both possibilities of enhancement and reduction of the luminescence energy.

Several changes to the setup might allow for an increased yield. The use of a highly purified liquid to decrease the cavitation threshold (in this setup cavitation noise was observed at $p_{A}=0.5$ bar and $\nu=6.3 \mathrm{kHz}$ ) would make it possible to drive the bubble with higher acoustic pressures. Further enhancement might be achieved by decreasing the ambient pressure. However, we expect with increased maximum bubble radius an increase in surface instability which might limit this approach. To gain further insight and to investigate the onset of surface instabilities the experiment should be equipped with a camera to monitor simultaneously the luminescence signal and the shape of the bubble prior to its collapse.

The author thanks T. Kurz for help with the experiment and W. Lauterborn, the members of the Cavitation and Sonoluminescence Group at the Drittes Physikalisches Institut, Göttingen, and H. Oguz for stimulating discussions. He further thanks W. Lauterborn for the use of the experimental equipment. This work has been supported by the Fraunhofer Gesellschaft.
[1] R.I. Nigmatulin, V.Sh. Shagapov, N.K. Vakhitova, and R.T. Lekhi, Jr., Dokl. Akad. Nauk 340, 37 (1995) [Phys. Dokl. 40, 122 (1995)].

[2] W.C. Moss, D.B. Clarke, J.W. White, and D.A. Young, Phys. Lett. A 211, 69 (1996).

[3] H.G. Flynn, U.S. Patent No. 4333796; S.J. Putterman, B.P. Barber, R.A. Hiller, and R.M.J. Lofstedt, U.S. Patent No. 5659173.

[4] J. Holzfuss, M. Rüggeberg, and R. Mettin, Phys. Rev. Lett. 81, 1961 (1998).

[5] F.D. Gaitan, L.A. Crum, C.C. Church, and R.A. Roy, J. Acoust. Soc. Am. 91, 3166 (1992); B.P. Barber, R.A. Hiller, R. Löeffstedt, S.J. Putterman, and K.R. Weninger, Phys. Rep. 281, 65 (1997).

[6] R.H. Cole, Underwater Explosions (Princeton University Press, Princeton, 1948); J.A. Cook, A.M. Gleeson, R.M. Roberts, and R.L. Rogers, J. Acoust. Soc. Am. 101, 1908 (1997); C. West and R. Howlett, Acustica 21, 112 (1969); W. Lauterborn, Appl. Phys. Lett. 21, 27 (1972).

[7] A. Vogel, S. Busch, and U. Parlitz, J. Acoust. Soc. Am. 100, 148 (1996).

[8] F.R. Gilmore (unpublished).
[9] C.D. Ohl, O. Lindau, and W. Lauterborn, in Sonochemistry and Sonoluminescence, edited by L.A. Crum, T.J. Mason, J.L. Reisse, and K.S. Suslick, Series C: Mathematical and Physical Sciences, Vol. 524 (Kluwer Academic Publishers, Dordrecht, 1999).

[10] E. Lax, Taschenbuch für Chemiker und Physiker (Springer Verlag, Berlin, 1967).

[11] C.D. Ohl, Zur Dynamik und Lumineszenz von Kavitationsblasen (Shaker Verlag, Aachen, Germany, 1999).

[12] Lord Rayleigh, Philos. Mag. 34, 94 (1917).

[13] An oscillating bubble can be described as a nonlinear oscillator acting as a hard spring during compression and like a soft spring during expansion.

[14] C.A. Sacchi, J. Opt. Soc. Am. B 8, 337 (1991).

[15] A. Philipp and W. Lauterborn, J. Fluid Mech. 361, 75 (1998).

[16] A boundary might cause aspherical bubble compression which influences light emission; see C.D. Ohl, O. Lindau, and W. Lauterborn, Phys. Rev. Lett. 80, 393 (1998).

[17] The duration of the SCBL (FWHM) in the absence of a sound field is found to increase with the maximum bubble size and is in the range of 3.5 to $9 \mathrm{~ns}$. 